\title{
Risk factors for periprocedural ischemic stroke following endovascular treatment of intracranial aneurysms
}

\author{
Yisen Zhang, Chao Wang, Zhongbin Tian, Wei Zhu, Wenqiang Li, Xinjian Yang, Jian Liu* and Ying Zhang ${ }^{*}$ (D)
}

\begin{abstract}
Background: The aim of this study was to comprehensively evaluate the risk factors of periprocedural ischemic stroke associated with endovascular treatment of intracranial aneurysms using a real-world database.

Methods: From August 2016 to March 2017, 167 patients were enrolled. Univariate analysis and multivariate logistic regression analysis were used to examine the risk factors for periprocedural ischemic stroke.

Results: Among the 167 cases, periprocedural ischemic stroke occurred in 20 cases (11.98\%). After univariate analysis, the ischemic group had a higher proportion of large ( $\geq 10 \mathrm{~mm}$ ) aneurysms than the control group ( $45.0 \% \mathrm{vs} .23 .1 \%, p$ $=0.036)$. The incidence of periprocedural ischemic stroke was higher in cases treated by flow diverter $(21.6 \%)$ or stentassisted coiling (11.8\%) than in cases treated by coiling only $(2.7 \%)$, and the differences were statistically significant $(p=$ 0.043). After multivariate logistic regression analysis, treatment modality was the independent risk factor for periprocedural ischemic stroke. Compared with the coiling-only procedure, flow diverter therapy was associated with a significantly higher rate of periprocedural ischemic stroke (OR 9.931; 95\% Cl 1.174-84.038; $p=0.035$ ).

Conclusions: Aneurysm size and treatment modality were associated with periprocedural ischemic stroke. Larger aneurysms were associated with increased risk of periprocedural ischemic stroke. Flow diverter therapy was associated with significantly more periprocedural ischemic stroke than the coiling procedure alone.
\end{abstract}

Keywords: Periprocedural ischemic stroke, Endovascular treatment, Intracranial aneurysms, Aneurysm size, Treatment modality

\section{Background}

In recent decades, endovascular treatment has become a major modality in the treatment of intracranial aneurysms, because the safety and efficacy have been demonstrated in numerous large clinical studies [1-3]. With the endovascular device improvements and increasing clinical experience, intracranial aneurysms could be treated by a more rational modality according to patient or aneurysm characteristics, which increased the

\footnotetext{
*Correspondence: jianliu_ns@163.com; yingzhang829@163.com Department of Interventional Neuroradiology, Beijing Neurosurgical Institute and Beijing Tian Tan Hospital, Capital Medical University, No.119, South 4th Ring West Road, Fengtai District, Beijing 100070, China
}

aneurysm occlusion rates [4]. For instance, the wideneck aneurysms $(\geq 4 \mathrm{~mm}$ or dome-neck ratio $\leq 2$ ) were usually treated by intracranial stent [5]. But for large or complex aneurysms, flow diverter therapy is the preferred treatment $[6,7]$.

However, endovascular treatment of intracranial aneurysm has the risk of neurological complications. Periprocedural ischemic stroke is the most common neurologic complication following endovascular treatment of intracranial aneurysms and is a major source of neurological morbidity and mortality $[8,9]$. A better understanding of the risk factors for periprocedural ischemic stroke is important and may prove beneficial in the

C C The Author(s). 2021 Open Access This article is licensed under a Creative Commons Attribution 4.0 International License, which permits use, sharing, adaptation, distribution and reproduction in any medium or format, as long as you give appropriate credit to the original author(s) and the source, provide a link to the Creative Commons licence, and indicate if changes were made. The images or other third party material in this article are included in the article's Creative Commons licence, unless indicated otherwise in a credit line to the material. If material is not included in the article's Creative Commons licence and your intended use is not permitted by statutory regulation or exceeds the permitted use, you will need to obtain permission directly from the copyright holder. To view a copy of this licence, visit http://creativecommons.org/licenses/by/4.0/ The Creative Commons Public Domain Dedication waiver (http://creativecommons.org/publicdomain/zero/1.0/) applies to the data made available in this article, unless otherwise stated in a credit line to the data. 
decision-making process of intracranial aneurysm management. However, limited studies have comprehensively evaluated the risks of periprocedural ischemic stroke associated with endovascular treatment of intracranial aneurysm using a real-world database. Therefore, the aim of this study was to evaluate the incidence and risk factors of periprocedural ischemic stroke following endovascular treatment using a real-world database. The clinical, aneurysmal, and procedural characteristics associated with periprocedural ischemic stroke were analyzed in this study.

\section{Methods}

\section{Patient selection}

This study is a retrospective study and the data on patients with intracranial saccular aneurysms treated by endovascular treatment were collected form a real-world database (the HARET study) [10]. Briefly, patients with a single unruptured intracranial saccular aneurysms who underwent endovascular treatment in our institute between August 2016 and March 2017 were enrolled in the current study. The exclusion criteria included multiple aneurysms, ruptured aneurysm, intracranial aneurysm with previous treatment, treatment by parent vessel occlusion, treatment by a covered stent, presence of a brain arteriovenous malformation, and presence of a dissecting aneurysm. Ultimately, 167 eligible patients with 167 aneurysms were enrolled in this study. The study was approved by our institutional review board. All patients or their relatives provided written informed consent during hospitalization. This retrospective case analysis study examined the variables associated with periprocedural ischemic stroke. Clinical, aneurysmal, and procedural factors were recorded and analyzed. These factors were as follows: age, sex, history of alcohol intake and cigarette smoking, hypertension, hyperlipidemia, previous ischemic comorbidity, aneurysm size (maximum size), shape (irregular: blebs, nipples or multiple lobes), sidewall/bifurcation, location (anterior/posterior circulation), distal aneurysm (at or distal to the Circle of Willis), and treatment modalities (including coiling only, stent-assisted coiling or flow diverter treatment). The occurrence of periprocedural ischemic stroke within 30 days of the endovascular treatment was also recorded, and the 167 eligible patients were divided into two groups (ischemic group and control group). All patients and aneurysms with periprocedural ischemic complications were included in the ischemic group. A major ischemic event was defined as an event lasting for more than 7 days, and a minor ischemic event was defined as an event that resolved within 7 days with no clinical sequelae [11]. All major ischemic events were included in the neurologic morbidity and mortality rates.

\section{Endovascular procedures}

For each endovascular procedure, the treatment strategy was discussed and chosen at the weekly peer-reviewed endovascular conference. Coiling alone was performed if the aneurysm neck was narrow $(<4 \mathrm{~mm}$ or dome-neck ratio $>2$ ). For wide-neck aneurysms $(\geq 4 \mathrm{~mm}$ or domeneck ratio $\leq 2$ ), the stent-assisted coiling technique was used. However, if the aneurysm was large or complex and therefore unsuitable for conventional endovascular treatment (coiling or stent-assisted coiling), the aneurysm was treated by a flow diverter. Endovascular treatment was performed under general anesthesia and systemic intravenous heparin. If stent-assisted coiling or flow diverter therapy was chosen, $100 \mathrm{mg} /$ day aspirin and $75 \mathrm{mg} /$ day clopidogrel were administered for at least 5 days before the procedure. Systemic intravenous heparin was administered in patients who underwent an endovascular procedure to maintain an activated clotting time between 250 and 300s, to prevent embolic events. Patients were treated with coiling, stent-assisted coiling, or flow diverter therapy as appropriate. After the procedure, patients treated with the conventional stent were given $75 \mathrm{mg} /$ day clopidogrel for 6 weeks and $100 \mathrm{mg} /$ day aspirin for 6 months, while patients treated with the flow diverter were given $75 \mathrm{mg} /$ day clopidogrel for 3 months and $100 \mathrm{mg} /$ day aspirin thereafter.

\section{Statistical analysis}

Data are presented as the mean \pm standard deviation for quantitative variables and frequency for qualitative variables. Risk factors associated with periprocedural ischemic complications were analyzed using an independent sample $t$-test, or a $\chi^{2}$ test applied as appropriate. Then, variables with $\mathrm{p}<0.20$ in the univariate logistic analysis were included in the multivariate logistic regression. Statistical significance was considered as $p<0.05$. Statistical analyses were performed using IBM SPSS Statistics for Windows, v.22.0 (IBM Corp., Armonk, NY, USA).

\section{Results}

\section{Clinical and aneurysmal characteristics}

In total, from August 2016 to March 2017, 167 patients with 167 aneurysms were included in this study. The patients consisted of 107 females and 60 males. The mean age of the patients was $56.17 \pm 10.13$ years. The mean size of the aneurysms was $8.10 \pm 5.50 \mathrm{~mm}$. Of the 167 aneurysms, 37 aneurysms were treated with coiling alone, 93 aneurysms with stent-assisted coiling and 37 aneurysms with flow diverter therapy.

\section{Periprocedural ischemic stroke}

In the current study, endovascular procedures were performed for the 167 patients with 167 aneurysms. Among the 167 patients, periprocedural ischemic stroke 
occurred in 20 procedures (11.98\%). The 20 periprocedural ischemic strokes consisted of 2 intraprocedural thrombus formations, 7 minor ischemic complications, and 11 major ischemic complications. Among the 20 periprocedural ischemic strokes, 7 cases presented with transient neurologic symptoms that resolved on discharge and 13 cases showed persistent neurologic morbidity (7.78\%). No mortality was observed.

\section{Risk factors for periprocedural ischemic stroke}

As shown in Table 1, after univariate analysis, aneurysm size was significantly correlated with occurrence of periprocedural ischemic stroke. The ischemic group had a higher proportion of large aneurysms $(\geq 10 \mathrm{~mm})$ than the control group $(45.0 \%$ vs. $23.1 \%, \mathrm{p}=0.036)$. Moreover, the incidence of periprocedural ischemic stroke was higher in cases treated by flow diverter therapy $(21.6 \%)$ or stent-assisted coiling (11.8\%) than in cases treated by coiling only $(2.7 \%)$, and the difference was statistically significant $(p=0.043)$ (Fig. 1$)$. The other clinical and aneurysmal factors examined (age, sex, smoking, drinking, hypertension, hyperlipidemia, previous ischemic stroke, shape, location, sidewall/bifurcation and distal/proximal to the circle of Willis) showed no significant differences between the ischemic and control groups $(p>0.05)$.

\section{Multivariate logistic regression analysis}

The multivariate logistic regression results are presented in Table 2. After multivariate logistic regression analysis, treatment modality was found to be the only independent risk factor for periprocedural ischemic stroke. Compared with the coiling-only procedure, flow diverter therapy was associated with a significantly higher rate of periprocedural ischemic stroke (OR 9.931; 95\% CI $1.174-84.038 ; p=0.035$ ).

\section{Discussion}

Periprocedural ischemic stroke is a major complication of endovascular treatment and is a major cause of

Table 1 Results from univariate statistical analysis for all variables

\begin{tabular}{|c|c|c|c|}
\hline Characteristics & Control group $(N=147)$ & Ischemic group $(N=20)$ & $P$ value \\
\hline Age (years) & $55.8 \pm 9.8$ & $58.7 \pm 12.4$ & 0.227 \\
\hline Sex (\%) & & & 0.556 \\
\hline Male & $54(36.7)$ & $6(30.0)$ & \\
\hline Female & $93(63.3)$ & $14(70.0)$ & \\
\hline Smoking (\%) & $21(14.3)$ & $4(20.0)$ & 0.735 \\
\hline Drinking (\%) & $20(13.6)$ & $1(5.0)$ & 0.466 \\
\hline Hypertension (\%) & $69(46.9)$ & $12(60.0)$ & 0.273 \\
\hline Hyperlipidemia & $42(28.6)$ & $5(25.0)$ & 0.739 \\
\hline Previous ischemic stroke & $23(15.6)$ & $3(15.0)$ & $>0.999$ \\
\hline Aneurysm size & & & 0.036 \\
\hline$<10 \mathrm{~mm}$ & $113(76.9)$ & $11(55.0)$ & \\
\hline$\geq 10 \mathrm{~mm}$ & $34(23.1)$ & $9(45.0)$ & \\
\hline Shape (\%) & & & 0.556 \\
\hline Regular & $93(63.3)$ & $14(70.0)$ & \\
\hline Irregular & $54(36.7)$ & $6(30.0)$ & \\
\hline Location (\%) & & & 0.479 \\
\hline Anterior circulation & $136(92.5)$ & $17(85.0)$ & \\
\hline Posterior circulation & $11(7.5)$ & $3(15.0)$ & \\
\hline Sidewall/bifurcation aneurysm (\%) & & & 0.691 \\
\hline Sidewall & $102(69.4)$ & $13(65.0)$ & \\
\hline Bifurcation & $45(30.6)$ & $7(35.0)$ & \\
\hline Distal aneurysm & $42(28.6)$ & $8(40.0)$ & 0.295 \\
\hline Treatment therapy (\%) & & & 0.043 \\
\hline Coiling & $36(24.5)$ & $1(5.0)$ & \\
\hline Stent-assisted coiling & $82(55.8)$ & $11(55.0)$ & \\
\hline Flow diverter & 29 (19.7) & $8(40.0)$ & \\
\hline
\end{tabular}




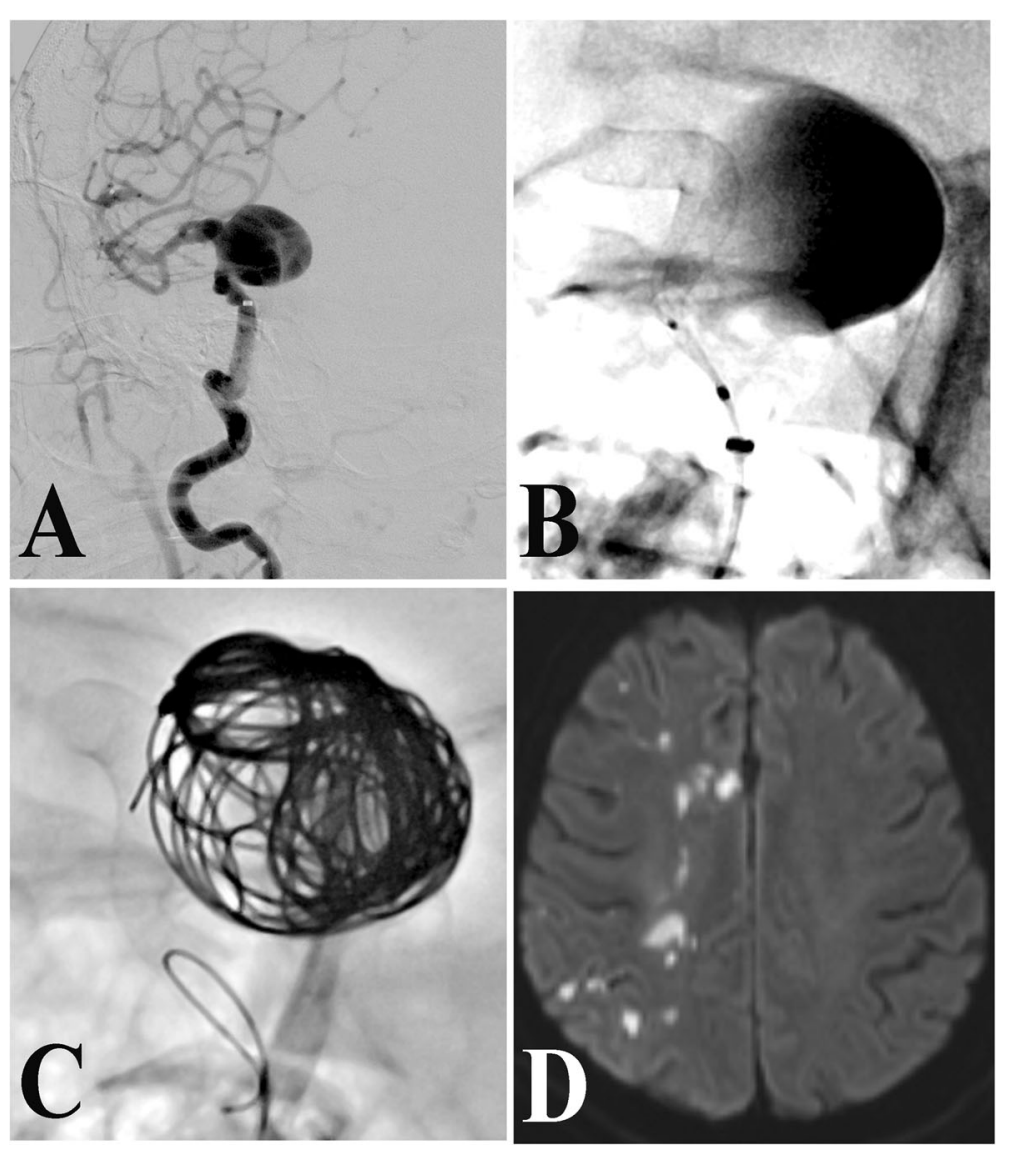

Fig. 1 A patient with a large aneurysm was treated by flow diverter with loose packing. A Digital subtraction angiogram showed the large aneurysm. B The pipeline embolization device was deployed. C Loose coils package was formed in the aneurysm sac. Five hours after the endovascular procedure, the patient presented with the weakness of the right limb. D Magnetic resonance imaging showed the infarct lesion

neurologic morbidity or even mortality. In the current study, using a real-world database, we comprehensively evaluated potential risk factors associated with periprocedural ischemic stroke after endovascular treatment of intracranial aneurysm. A main finding is that aneurysm size and endovascular treatment modality were associated with the occurrence of periprocedural ischemic stroke. Treatment modality was the only independent risk factor for periprocedural ischemic stroke in this

Table 2 Multivariate logistic regression results

\begin{tabular}{|c|c|c|}
\hline Variables & $\mathrm{OR}, 95 \% \mathrm{Cl}$ & $p$ value \\
\hline \multicolumn{3}{|l|}{ Aneurysm size } \\
\hline$<10 \mathrm{~mm}$ & & Ref \\
\hline$\geq 10 \mathrm{~mm}$ & $1.773(0.587,5.356)$ & 0.310 \\
\hline \multicolumn{3}{|l|}{ Treatment therapy } \\
\hline Coiling & & Ref. \\
\hline Stent-assisted coiling & $4.829(0.601,38.822)$ & 0.139 \\
\hline Flow diverter & $9.931(1.174,84.038)$ & 0.035 \\
\hline
\end{tabular}

study. We believe the results of this study could provide a beneficial reference for physicians.

Larger aneurysms are associated with increased risk of periprocedural ischemic stroke [12]. Intra-aneurysmal clot is more frequent in larger aneurysms before endovascular treatment, and larger aneurysms are more likely to have residual flow within the coil mass than small aneurysms [13-15]. Furthermore, for stent deployment, large size aneurysms can make it difficult to achieve good wall apposition, thus increasing the risk of periprocedural ischemic stroke [8]. The present study also found that the ischemic group had a higher proportion of large aneurysms than the control group.

Treatment modality was also associated with periprocedural ischemic stroke after endovascular treatment. Compared with coiling treatment alone, the risk of ischemic events is particularly high after stent-assisted coiling or flow diverter deployment, which may be due to the thrombogenicity of intra-arterial devices, longer procedure times, and the complexity of the procedures [16]. Moreover, the disturbed flow across the aneurysm neck with ingress and egress through the 
stent lumen may also increase the risk of periprocedural ischemic stroke [17]. In this study, treatment modality was the only independent risk factor for periprocedural ischemic stroke.

Periprocedural ischemic stroke mainly results from embolic events during endovascular treatment of intracranial aneurysms, such as the stent wall thrombus, original thrombus, or fresh clot migrating distally during the procedure [18]. Because platelets are the primary component of thrombi, inhibition of platelet reactivity can reduce the occurrence of ischemic complications, especially for stent deployment therapy [14]. Therefore, dual antiplatelet therapy (aspirin and clopidogrel) is widely accepted as the standard protocol to decrease the risk of ischemic complications for intracranial aneurysms treated with stents [17]. However, many patients still suffer periprocedural ischemic stroke after stent deployment, even though the standard antiplatelet medication protocol is applied [18]. Therefore, individualized antiplatelet therapy still needs to be investigated.

There are several limitations of our study. First, a limited number of cases, retrospective design and patient selection bias may limit the generalization of the results. Also, computerized tomography and magnetic resonance are not part of the routine clinical examination after endovascular treatment, so some asymptomatic ischemic complications may not have been detected. Finally, longterm follow-up results and the role of platelet function monitoring were not included in the study; these will be reported in future work.

\section{Conclusions}

Ischemic stroke was the most common procedurerelated complication of endovascular treatment. Larger aneurysms increased the risk of periprocedural ischemic stroke. Treatment modality was the independent risk factor of periprocedural ischemic stroke found in this study. Flow diverter therapy resulted in significantly more periprocedural ischemic stroke than the coiling procedure alone.

\section{Abbreviations}

HARET: Haemodynamic analysis for recanalisation of intracranial aneurysms after endovascular treatment; OR: Odds ratio; Cl: Confidence interval

\section{Acknowledgements}

Not applicable.

\section{Authors' contributions}

Yisen Zhang performed the statistical analysis and the manuscript writing Chao Wang, Zhongbin Tian, Wei Zhu, and Wenqiang Li acquired the data. Xinjian Yang and Jian Liu analyzed and interpreted the data. Ying Zhang and Jian Liu conceived and designed the research and handled funding and supervision. The authors read and approved the final manuscript.

\section{Funding}

This work was supported by National Key Research and Development Plan of China (grant number: 2016YFC1300800), the National Natural Science
Foundation of China (grant numbers: 8207071595, 81801156, 81801158 and 81671139), the Special Research Project for Capital Health Development (grant number: 2018-4-1077), and Beijing Hospitals Authority Youth Programme (code: QML20190503).

Availability of data and materials

In addition to the data published within this article, anonymized data can be obtained by request by any qualified investigator.

\section{Declarations}

Ethics approval and consent to participate

The ethics committee of Beijing Tian Tan Hospital approved this study (KYSQ 2021-138-01). All procedures performed in studies involving human participants were in accordance with the ethical standards of the institutional and/ or national research committee and with the 1964 Helsinki Declaration and its later amendments or comparable ethical standards. Informed consent was obtained from all individual participants included in the study.

\section{Consent for publication}

Not applicable.

\section{Competing interests}

We declare that we have no conflict of interest.

Received: 10 January 2021 Accepted: 2 August 2021

Published online: 23 August 2021

\section{References}

1. Molyneux AJ, Kerr RS, Yu LM, Clarke M, Sneade M, Yarnold JA, et al. International subarachnoid aneurysm trial (ISAT) of neurosurgical clipping versus endovascular coiling in 2143 patients with ruptured intracranial aneurysms: a randomised comparison of effects on survival, dependency, seizures, rebleeding, subgroups, and aneurysm occlusion. Lancet. 2005; 366(9488):809-17. https://doi.org/10.1016/S0140-6736(05)67214-5.

2. Molyneux AJ, Birks J, Clarke A, Sneade M, Kerr RSC. The durability of endovascular coiling versus neurosurgical clipping of ruptured cerebral aneurysms: 18 year follow-up of the UK cohort of the International Subarachnoid Aneurysm Trial (ISAT). Lancet. 2015;385(9969):691-7. https:// doi.org/10.1016/S0140-6736(14)60975-2.

3. Gonda DD, Khalessi AA, McCutcheon BA, et al. Long-term follow-up of unruptured intracranial aneurysms repaired in California. J Neurosurg. 2014; 120(6):1349-57. https://doi.org/10.3171/2014.3.JNS131159.

4. Naggara ON, Lecler A, Oppenheim C, Meder JF, Raymond J. Endovascular treatment of intracranial unruptured aneurysms: a systematic review of the literature on safety with emphasis on subgroup analyses. Radiology. 2012; 263(3):828-35. https://doi.org/10.1148/radiol.12112114.

5. Yang $H$, Sun $Y$, Jiang $Y, L v X$, Zhao $Y$, Li Y, et al. Comparison of stent-assisted coiling vs coiling alone in 563 intracranial aneurysms: safety and efficacy at a high-volume center. Neurosurgery. 2015;77(2):241-7; discussion 247. https://doi.org/10.1227/NEU.0000000000000765.

6. Zhang Y, Zhou Y, Yang P, Liu J, Xu Y, Hong B, et al. Comparison of the flow diverter and stent-assisted coiling in large and giant aneurysms: safety and efficacy based on a propensity score-matched analysis. Eur Radiol. 2016; 26(7):2369-77. https://doi.org/10.1007/s00330-015-4052-1.

7. Chalouhi N, Tjoumakaris S, Starke RM, Gonzalez LF, Randazzo C, Hasan D, et al. Comparison of flow diversion and coiling in large unruptured intracranial saccular aneurysms. Stroke. 2013;44(8):2150-4. https://doi.org/1 0.1161/STROKEAHA.113.001785

8. Brinjikji W, Lanzino G, Cloft HJ, Siddiqui AH, Boccardi E, Cekirge S, et al. Risk Factors for Ischemic Complications following Pipeline Embolization Device Treatment of Intracranial Aneurysms: Results from the IntrePED Study. AJNR Am J Neuroradiol. 2016;37(9):1673-8. https://doi.org/10.3174/ajnr.A4807.

9. Park HK, Horowitz M, Jungreis C, Genevro J, Koebbe C, Levy E, et al. Periprocedural morbidity and mortality associated with endovascular treatment of intracranial aneurysms. AJNR Am J Neuroradiol. 2005;26(3): 506-14

10. Liu J, Zhang Y, Wang A, Zhang Y, Li Y, Yang X. Haemodynamic analysis for recanalisation of intracranial aneurysms after endovascular treatment: an observational registry study in China. BMJ Open. 2017;7(5):e014261. https:// doi.org/10.1136/bmjopen-2016-014261. 
11. Kallmes DF, Hanel R, Lopes D, Boccardi E, Bonafe A, Cekirge S, et al. International retrospective study of the pipeline embolization device: a multicenter aneurysm treatment study. AJNR Am J Neuroradiol. 2015;36(1): 108-15. https://doi.org/10.3174/ajnr.A4111.

12. Pierot L, Spelle L, Vitry F. Immediate clinical outcome of patients harboring unruptured intracranial aneurysms treated by endovascular approach: results of the ATENA study. Stroke. 2008;39(9):2497-504. https://doi.org/1 $0.1161 /$ STROKEAHA.107.512756.

13. Derdeyn CP, Cross DT 3rd, Moran CJ, et al. Postprocedure ischemic events after treatment of intracranial aneurysms with Guglielmi detachable coils. Neurosurg. 2002;96(5):837-43. https://doi.org/10.3171/jns.2002.96.5.0837.

14. Ihn YK, Shin SH, Baik SK, Choi IS. Complications of endovascular treatment for intracranial aneurysms: management and prevention. Interv Neuroradiol. 2018;24(3):237-45. https://doi.org/10.1177/1591019918758493.

15. Pierot L, Cognard C, Anxionnat R, Ricolfi F, for the CLARITY Investigators. Ruptured intracranial aneurysms: factors affecting the rate and outcome of endovascular treatment complications in a series of 782 patients (CLARITY study). Radiology. 2010;256(3):916-23. https://doi.org/10.1148/radiol.10092209.

16. Adeeb N, Griessenauer CJ, Moore JM, Foreman PM, Shallwani H, MotieiLangroudi $R$, et al. Ischemic stroke after treatment of intraprocedural thrombosis during stent-assisted coiling and flow diversion. Stroke. 2017; 48(4):1098-100. https://doi.org/10.1161/STROKEAHA.116.016521.

17. Ge H, Yang H, Ren H, Li W, Lv X, Jin H, et al. Association of thrombelastographic parameters with complications in patients with intracranial aneurysm after stent placement. World Neurosurg. 2019;127: e30-8. https://doi.org/10.1016/j.wneu.2019.02.007.

18. Yang $H$, Li Y, Jiang Y. Insufficient platelet inhibition and thromboembolic complications in patients with intracranial aneurysms after stent placement. J Neurosurg. 2016;125(2):247-53. https://doi.org/10.3171/2015.6.JNS1511.

Ready to submit your research? Choose BMC and benefit from:

- fast, convenient online submission

- thorough peer review by experienced researchers in your field

- rapid publication on acceptance

- support for research data, including large and complex data types

- gold Open Access which fosters wider collaboration and increased citations

- maximum visibility for your research: over $100 \mathrm{M}$ website views per year

At $\mathrm{BMC}$, research is always in progress.

Learn more biomedcentral.com/submissions 